\title{
Expert System For Diagnosis Pest And Disease In Fruit Plants
}

\author{
Satrio Dewanto ${ }^{1}$ and Jonathan Lukas ${ }^{1}$ \\ ${ }^{1}$ Universitas Bina Nusantara, Computer Engineering Department, Jakarta, Indonesia
}

\begin{abstract}
This paper discussed the development of an expert system to diagnose pests and diseases on fruit plants. Rule base method was used to store the knowledge from experts and literatures. Control technique using backward chain and started from the symptoms to get conclusions about the pests and diseases that occur. Development of the system has been performed using software Corvid Exsys developed by Exsys company. Results showed that the development of this expert system can be used to assist users in identifying the type of pests and diseases on fruit plants. Further development and possibility of using internet for this system are proposed.
\end{abstract}

\section{Introduction}

As a tropical country, Indonesia is very rich in fruits. It is unfortunate that the consumption of Indonesian society is still relatively low compared to countries that do not have the resources as a producer of fruit. Especially considering that fruits contain nutrients that are essential for health. Along with the increasing awareness of healthy living, encourage people to increase consumption of fruits, as a habit that is based on the principle of back to nature. Namely, a lifestyle utilizing natural fresh ingredients as much as possible for daily life.

Existing climate has made Indonesia as a heaven for the availability of various types of tropical fruit. This led to Indonesia being called as tropical fruit paradise. Fruit development policy in Indonesia is based on consideration of economic value, comparative advantage, agro-climatic suitability, agribusiness and agro-industries. Nowadays development priorities of fruits can be divided into national priorities, including: orange, mango, rambutan, durian and bananas, and local priority which include: mangosteen, duku, lychee, longan and passion fruit.

Problems that exist in Indonesian fruits are low quality, quantity and continuity as well as consistency of taste leads to low competitiveness of the national fruit. As one of the causes is pests and diseases. Pest and disease control is a very important part in improving the quality and quantity of fruit production. In general, farmers and private industry engaged in agribusiness fruits still have not mastered the details on plant pests and diseases and how to control [1]. This research will develop an expert system software to diagnose pests and diseases of fruit plants. By using this system, users can identify the type of disease in fruit plants with ease.

\section{Pests And Plant Diseases.}

The purpose of human to do cultivation is to meet the needs of growing food along with the increase of population. In addition the plant also serves as a provider of oxygen to the human respiratory system as well as the aesthetic and the beauty that can be enjoyed by humans. Therefore, research on plant cultivation continues to be done to get a high yielding plant that is superior, responsive to fertilization and resistance to pests and plant diseases. Studies on the pests control and diseases of plants grown rapidly along with the man's efforts to get optimal results from its cultivated plants. Pest and disease attack and destroy crop cultivation efforts and result in reduced quality and quantity of the results obtained.

Problems of pests and plant diseases are the main obstacles in increasing agricultural production. An estimated one-third of the world's agricultural production has been marred by more than 20,000 species of destructive organisms including pests and plant diseases. Damage occurs, both on the field during the cultivation process and warehouse storage. These conditions will significantly affect the income of farmers and the world's food supply. Destructive organisms covers all forms of life that can destroy plants and classified into 3 groups. The first group is the pest, ie animal or nuisance animals and destroying plants such as insects, mollusks and mammals. The second category is a disease caused by micro bodies such as fungi, bacteria and viruses. 
The third is the weed plants that are not expected its presence on an agricultural area [2]. Pests and plant diseases are still a complicated issue for owners of the plants, especially for those who do not have the basic knowledge of crop cultivation. The problem becomes more complex due to many types of pests and plant diseases. To differentiate the cause of damage to crops, long enough experience are needed so there is no error in concluding the cause in order to take the right decision in an effort to control.

\section{Expert System}

According to Turban (1992 ) expert system is a system that uses human knowledge in a computer to solve a problem that requires human expertise [ 3 ]. The basic concept of the expert system is expertise, expert, expertise transfer, draw conclusions, and the ability to explain the rule. Expertise is broad and specific knowledge and gained from the exercise, read, experience. The purpose of the expert system is moving into the expertise of the computer and then to others, especially not an expert. The transfer process involves four activities, namely the collection of knowledge , knowledge representation, inference of rules which exist and the transfer of knowledge to users .

The uniqueness of the expert system is the ability to reason. All the knowledge stored in the knowledge base and the program has the ability to access into a database and programmed computers in order to draw conclusions. Conclusions resulting in a component called the inference engine that includes procedures for solving problems . Most commercial expert system rule base method using the knowledge stored in the form of rule - shaped if-then rule. The uniqueness of the system is its ability to provide expert advice or recommendation and even justify why an action should not be recommended .

Structure of the expert system in general may contain several components, among others :

a. Knowledge Acquisition Subsystem : accumulation, transfer and transformation of expertise in solving problems stemming from experts or knowledge sources are documented .

b. Knowledge Base : Contains the knowledge used to understand, formulate and solve the problem .

c. Inference Engine : This is the brain of an expert system which is usually a program that has the ability to formulate final conclusions .

d . Blackboard ( Workplace ) : area where the memory is being used on the set as a database to describe the problem indicated by the input data. Also used to record the results, hypotheses and decisions .

e . User Interface : Language processor that is used for communication between the user and the computer .

f . Explanation Subsystem ( Justifier ) : The ability to explain the behavior of an interactive expert system .

g. Knowledge Refining System : The system can analyze and use knowledge, learn from the knowledge and improvisation for the upcoming consultations conducted as befits an expert.
In addition to the above components, there are still additional components which are the user and not an expert that requires advice or training.

\section{Methodology}

Pest and disease attack and destroy crop cultivation efforts and result in reduced quality and quantity of the results obtained. With the acquisition of knowledge from experts and literature can be designed in the form of a computer program package expert system to help growers of fruits and investors in the field of agro-industry in identifying pests and diseases of fruit crops and how to control without having to consult directly with experts.

Expert system will be developed using Exsys software can be modeled by a block diagram as shown below.

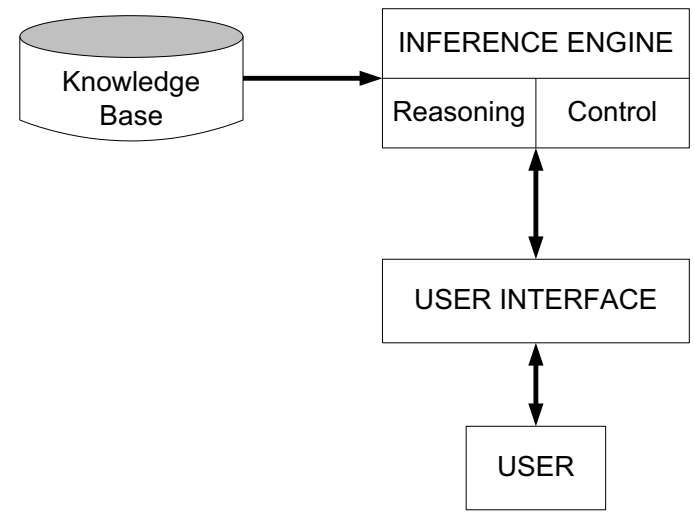

Fig. 1 : Block diagram of expert system.

Knowledge base regarding pests and diseases of plants obtained from experts and literatures as well as internet sources will be transferred into a computer using software tools Exsys. Exsys software is a development tool of expert system that combines a powerful rule editor with a flexible visual interface of decision trees and inference engine. Applications developed to run itself as a stand-alone or incorporated into the website.

Knowledge will be represented using a decision tree and then be converted into the form of if-then rules that are stored in the knowledge base. Inference engine is the most important component of an expert system. In the inference engine the process occurs to manipulate and directing rules, models and facts stored in the knowledge base, in order to get solutions or conclusions [4]. Control techniques used is the backwards chaining.

Backward chaining is referred to as Goal Driven. When run in a backward chaining mode, the system always has a "goal list" of the Goals it is trying to achieve. This list is fundamental to backward chaining. Each item in the list is a variable. This could be a numeric variable such as a weight or cost, a multiple choice list or other types of variables that can be assigned a value.

The top on the list is the one that the inference engine is actively trying to find a value for. To get that value, the inference engine will try to find rules that 
could be used to derive, or set a value for the variable. If such a rule is found, the IF conditions in the rule are tested to determine if the rule is true. If there is enough data already in the system, the IF conditions of the rule may be able to be immediately determined to true or false. If they are true, the rule can be used to set the value for the top variable on the Goal List. If the rule is determined to be false, the rule is discarded. However, if the there is not enough data to tell if the rule is true or false, the inference engine determines what additional information it would need to evaluate the rule.

This results in the system putting a new variable on the top of the Goal List. This become the new active "Goal" to be evaluated. This variable is needed to evaluate the rule being tested, but for the moment, that rule is ignored and the inference engined concentrates on finding a value for the new top variable on the Goal List.

As variables have values assigned, they are removed from the Goal List. The next variable in the list becomes the new top variable being actively worked on. This process continues untill all relevant variables have been assigned values, including the initial variable that started the process. Once that starting variables is assigned a value and removed from the list, the process is complete.

Users may consult to gain knowledge and advice as well as expert advice or justification to use dialogue with the computer through a user interface. In the final stage will be the implementation results of the above formulation using software selected and testing to see if the system is made to represent an expert.

\section{Result and Discussion}

Knowledge base that has been compiled in the form of ifthen rules by using the Exsys software will be used to draw the conclusions.

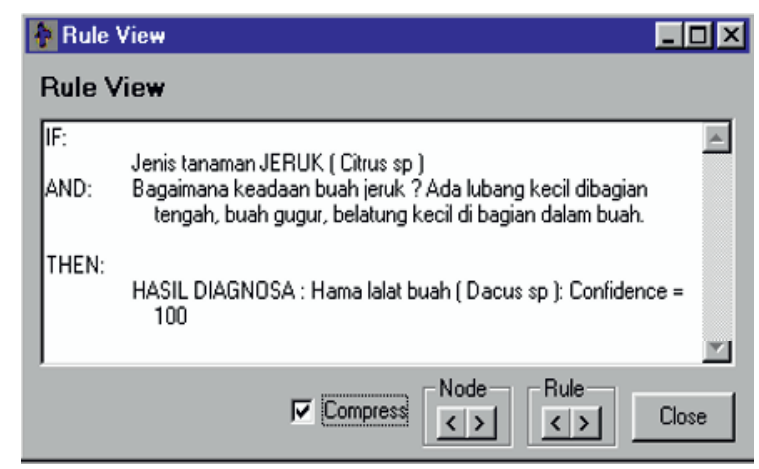

Fig. 2: display of if-then rule.

The system developed by Exsys software will ask interactively to the user about the value of the variables used in the if-then rules. Variables used are static lists and confidence. Based on input from the user, the software can produce a goal from this process in the form of pests and diseases that occur in fruit crops. Decision-making logic on Exsys is built using "nodes". A point (node) is a statement in the IF or THEN to a rule. Nodes can be used to create logic tree diagram so that the rule-path easily analyzed. Track rules using a tree diagram on a system that is made can be seen below.

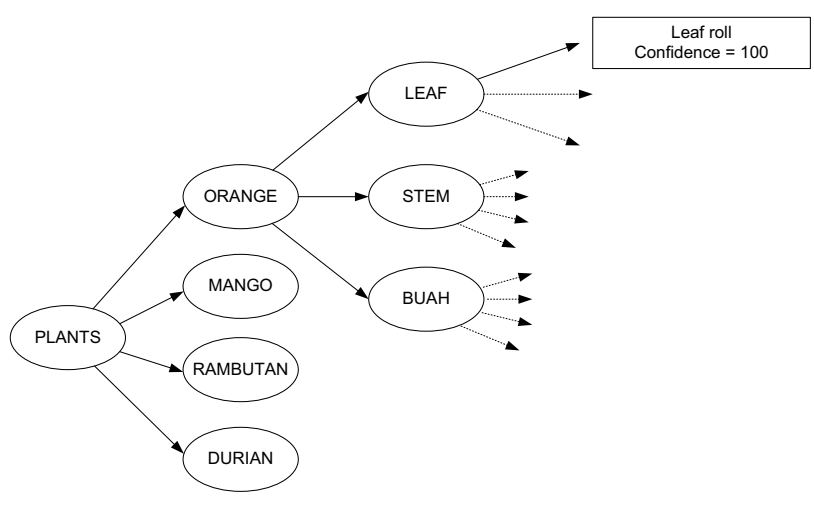

Fig. 3 : Tree Diagram for rule-path.

Diagnosis can only be made based on the symptoms that have been observed [5]. User usually only know the common symptoms such as leaf color change, fall of leaf / fruit and the system must be able to explain this phenomenon by considering all causes [6]. The cause of the symptoms that occur may be more than one so that it appears uncertain. Problem of uncertainty in the existing variable is handled using confidence variable that has a value between $0-100$. Overall confidence value is calculated using a formula that is used in the system sum method. In the sum method confidence values will be totaled. Positive value will increase the certainty / confidence while a negative value will reduce certainty.

Initial display gives the user a choice about the type of plants you want to diagnosed on the case. Fruit species that have been made are durian, mango, rambutan and oranges. Next appearance in the form of questions about the symptoms occurs in the leaves and can be seen in the picture below for mango plant.

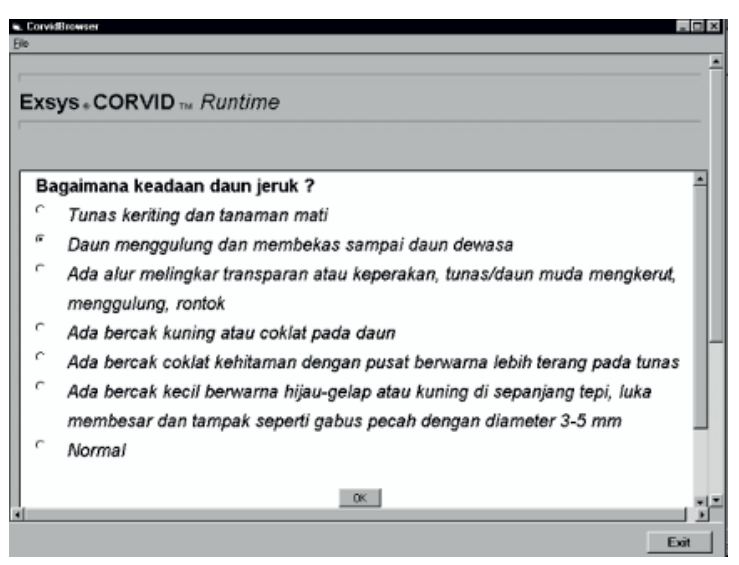

Fig. 4 : Symptoms of oranges leaf

After the user selects an answer to the questions above, then the next questions arise for symptoms on stems, twigs and fruit. On each display, the user simply choose one of several answers based on the symptoms 
that users know. Based on several questions and answers of the user, the expert system can perform reasoning to provide diagnosis of the pests and diseases experienced by plants and their fruit in the form of advice and recommendations how to control pests and diseases.

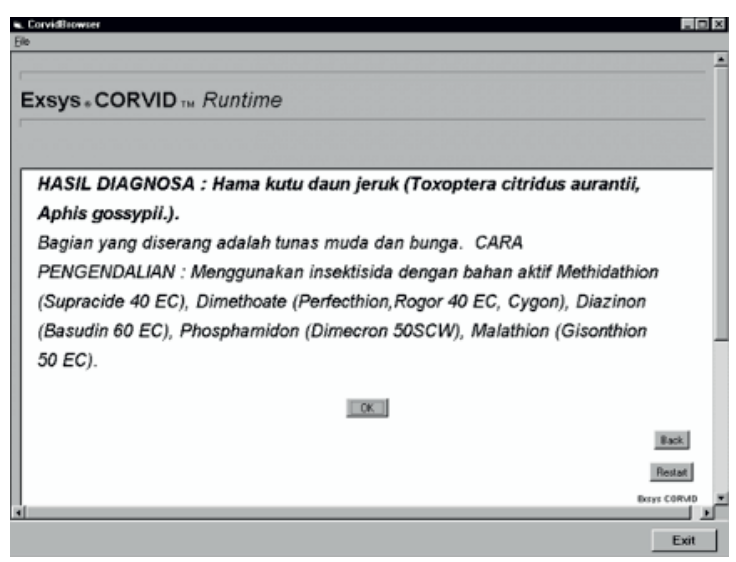

Fig. 5 : Result of diagnosis and suggestion.

After several tries of this expert system can be obtained that the output in the form of diagnosis and suggestions provided are in accordance with the knowledge obtained from the literatures. Furthert test and evaluation of this expert system is needed in the laboratory and field testing to know the benefits of this system.

\section{Conclusion}

Expert system that was developed using the if-then rules can be used for drawing conclusions on the issue of pests and diseases of fruit plants. The system has a mechanism of drawing conclusions based on knowledge that has been stored in the knowledge base and interactive dialogue between users and systems.

This expert system can be used by the user and beneficial for the user, especially where the presence of experts difficult to find and a reference book on pests and plant diseases are difficult to obtain. The user can easily operate the system and obtain results quickly, based on the known symptoms of the plant without the need for direct consultation with a specialist on pest and plant diseases.

For the system to be more efficient and effective, it is necessary to use hardware with better specs. Figure of pests or plant diseases should be displayed when the user's mouse is on the options provided. Exsys have the ability to run using the internet so that the expert system would be better if it can be uploaded on the Internet so that users from all over area can utilize this system.

System development needs to be done to increase the number of fruit plants that can be diagnosed, especially fruits that become a national priority for the chance to be cultivated and exported. Verification and validation by experts and practitioners need to be done to improve the knowledge base.

\section{References}

1 Teknologi Tepat Guna,tentang budi daya pertanian, http://www.iptek.net.id/ind/

2 Joesi, E \& Novizan, 2002, Mengendalikan hama dan penyakit tanaman, PT Agro Media Pustaka, Depok.

3 Turban,Efraim, 1992, Expert system and applied artificial intelligence, Macmillan Publishing Company, Singapore.

4 Marimin. 2002. Teori dan aplikasi sistem pakar dalam teknologi manajerial, IPB Press, Bogor.

5 Thomas, M.B. et.al, 1997, Two computer-based diagnostic expert systems for disease, insect pests, and physiological disorders of citrus and selected tropical fruit crops. HortTechnology 7:293-298.

6 Kemp R.H., Stewart T.M. and Boorman A., 1989: An expert system for diagnosis of pests, diseases, and disorders in apple crops. New Zealand Journal of Crop and Horticultural Science. 17: 89-96. 